\title{
RELIABILITY OF SAMPLING INSPECTION SCHEMES APPLIED TO REPLACEMENT STEAM GENERATORS
}

\author{
Guy Roussel \\ Association Vinçotte Nuclear \\ Rue Walcourt 148 Walcourtstraat, B-1070 Brussels \\ Tel.: +32.2.5280.246, Fax.: +32.2.5280.102, E-mail: gr@avn.be \\ Leon Cizelj \\ Institut "Jožef Stefan" \\ Jamova 39, SI-1000 Ljubljana, Slovenia \\ Tel.: +386.1.5885.215, Fax.: + 386.1.5885.377, E-mal: Leon.Cizelj@,ijs.si
}

\begin{abstract}
The basis for determining the size of the random sample of tubes to be inspected in replacement steam generators is revisited in this paper. A procedure to estimate the maximum number of defective tubes left in the steam generator after no defective tubes have been detected in the randomly selected inspection sample is proposed. A Bayesian estimation is used to obtain closed-form solutions for uniform, triangular and binomial prior densities describing the number of failed tubes in steam generators.
\end{abstract}

It is shown that the particular way of selecting the random inspection sample (e.g., one sample from both SG, one sample from each SG, etc.) does not affect the results of the inspection and also the information obtained about the state of the uninspected tubing, as long as the inspected steam generators belong to the same population. Numerical examples further demonstrate two possible states of the knowledge existing before the inspection of the tubing. First, virtually no knowledge about the state of the steam generator tubing before the inspection is modeled using uniform and triangular prior densities. It is shown that the knowledge about the uninspected part of the tubing strongly depends on the size of the sample inspected. Further, even small inspection samples may significantly improve our knowledge about the uninspected part. On the other hand, rather strong belief on the state of the tubing prior to the inspection is modeled using binomial prior density. In this case, the knowledge about the uninspected part of the tubing is virtually independent on the size of the sample. Furthermore, it is shown qualitatively and quantitatively that such inspection brings no additional knowledge on the uninspected part of the tubing.

\section{INTRODUCTION}

In Pressurized Water Reactors, a program of periodic in-service inspection of steam generator tubes is set up to monitor the integrity of the tubes. The in-service inspection is performed using non-destructive examination techniques, e.g., eddy current testing. Especially in replaced steam generators, not all the tubes are inspected but the inspection is limited to a sample of tubes. Therefore the objective of the in-service inspection is to provide reasonable insurance of steam generator tubing integrity. Consequently, the concern is the level of confidence that can be placed in the estimated knowledge about the whole population of the steam generator tubes from the information obtained from the examination of a sample of a limited size. 
The usual procedure [1] serving as a basis for determining the percentage of tubes sampled is to provide, by means of a statistical analysis, an equation that relates the percentage of tubes sampled, the number of degraded tubes present in the total population, and the probability (confidence level) that at least one defective tube will be sampled during the examination. For instance, this equation allows determining the number of defective tubes required to provide a 90 percent level of confidence that at least one defective tube will be sampled for a given percentage of tubes sampled.

In replacement steam generators, the number of defective tubes is expected to be very low. Indeed, the use of tube material showing less sensitivity to stress corrosion cracking as well as improvements in design and fabrication make the occurrence of degradation mechanism less likely. The usual sample may then simply be too small to include a defective tube. Hence, the basis for determining the percentage of tubes sampled is revisited in this paper and is aiming at the estimation of the number of defective tubes in the total population given that no defective tubes have been detected in an inspection sample. Otherwise stated, we are estimating the failure probability from data that contain zero defects. To this end a Bayesian estimation procedure is used.

Numerical solution of resulting Bayesian equation has been reported in [2]. In this paper, the relation between the estimated number of defective tubes at a given confidence level and the size of the sample given that no degraded tubes have been detected in the sample is derived in a closed-form for a selected set of prior distributions. A realistic numerical example is provided to arrive at interesting quantitative conclusions.

The failure probability of defective tubes steam generators is not discussed in this paper. Rather extensive literature exists on this matter - see for example [3], [4], [5] and references therein.

\section{MATHEMATICAL}

It is our intention to arrive at closed-form expressions relating the fact that the inspection of a small sample selected randomly from the population showed exactly zero defects, with the probability of having certain number of defective tubes in the finite population.

To this end, some closed-form solutions are derived using the Bayesian probability theory. Also, some approximations found in the literature are discussed.

\subsection{Basic relations}

Consider a lot of $N$ units of the same type. It is expected that there are a few defective units in the lot. Before inspection, however, there is no known reason to distinguish among different units as far as their individual plausibility to be defective is concerned.

Random sampling of $n$ units from the lot may be considered as a random drawing without replacement of $n$ units from the lot. Put $S_{n}$ the number of defective units in a random sample of size $n$. The case where $n=N$ means that the sampling without replacement has been performed until all units have been drawn and hence $S_{N}$ is the number of defective units contained in the lot.

In the case where the lot is known to include $k$ defective tubes, the probability of $l$ defective units among any random sample of size $n$ follows the hypergeometric distribution and is given by 


$$
P\left(S_{n}=l \mid S_{N}=k\right)=\frac{\left(\begin{array}{l}
k \\
l
\end{array}\right)\left(\begin{array}{c}
N-k \\
n-l
\end{array}\right)}{\left(\begin{array}{l}
N \\
n
\end{array}\right)}=h(l, n, k, N) .
$$

In the case where the composition of the population is unknown, the probability of $l$ defective tubes among any random sample of size $n$ is given by the mixture of hypergeometric probabilities by application of the rule of total probabilities

$$
P\left(S_{n}=l\right)=\sum_{k=l}^{N-n+l} P\left(S_{n}=l \mid S_{N}=k\right) P\left(S_{N}=k\right)=\sum_{k=l}^{N-n+l} h(l, n, k, N) P\left(S_{N}=k\right) .
$$

Applying Bayes' theorem,

$$
P\left(S_{N}=k \mid S_{n}=l\right)=\frac{P\left(S_{n}=l \mid S_{N}=k\right) P\left(S_{N}=k\right)}{P\left(S_{n}=l\right)}=\frac{h(l, n, k, N) P\left(S_{N}=k\right)}{\sum_{k^{\prime}=l}^{N-n+l} h\left(l, n, k^{\prime}, N\right) P\left(S_{N}=k^{\prime}\right)} .
$$

Our study, based on Eq.( 3), will be developed in the frame of a subjectivist interpretation of probability, namely the Bayesian approach of probability. A characteristic of subjective probability is that any unknown quantity is treated as a random variable and uncertainty about it is expressed by means of a probability distribution. In the Bayesian probability theory, the probability of an event describes the observer's degree of belief on the occurrence of the event, related with a personal state of information.

The Bayes' theorem, written in terms of discrete probability function models, takes the form:

$$
P(k \mid l)=\frac{h(l \mid k) P(k)}{\sum_{k} h(l \mid k) P(k)} .
$$

In the Bayesian approach, the prior belief about the probability of $k$ is quantified by a probability distribution, the prior distribution of $k$, i.e., $P(k)$. Data $l$ are then collected, and the likelihood function $h(l \mid k)$ is constructed. Finally the posterior distribution $P(k \mid l)$ is constructed, by combining the prior distribution $P(k)$ and the likelihood function $h(l \mid k)$ through Bayes' theorem. The posterior distribution shows the updated belief about the values of the probability that accounts for the observed data. The integral in the denominator ensures that the right hand side of the equation is properly scaled. In any case, it is just a constant that is independent of the values of the parameter $k$.

When the parameter $k$ is discrete, the simplest way is to choose, as a prior distribution, a function that combines neatly with the likelihood to give a posterior that can be evaluated by relatively simple formulas. However, often there is no closed-form expression for the integral in the denominator and the posterior density function must be calculated numerically.

\subsection{Posterior Probabilities of Defective Tubes}

While performing the inservice inspection of the steam generator tubes, the question may arise whether if it is sufficient to inspect a random sample from any steam generator or the inspection samples should be taken separately from each steam generator. The answer to this question is attempted below, addressing three particular cases:

- One sample from all steam generators

- One sample from one steam generator

- Two samples from two steam generators 
For the sake of simplicity, a two-loop plant has been considered. The conclusions may be nevertheless extended to an n-loop plant.

Some additional assumptions have also been made:

1. all steam generators are performing in a like manner;

2. only one flaw may affect a steam generator tube;

3. the samples are selected on a random basis;

4. the probability of detection of flaws with size larger than the detection threshold is 1 .

\subsubsection{One sample from all steam generators}

Consider first the simplest case where one sample is selected randomly from both steam generators. It is convenient to choose the sample size of $2 n$ to be taken from the total population of $2 N$ tubes.

Let $S_{2 n}$ be the number of defective tubes in a random sample of size $2 n$ taken from both steam generators. Hence, $S_{2 N}$ is the number of defective tubes in the total population.

Applying Eq. ( 3), the probability of having $l$ defective tubes in the total population is given by

$$
P\left(S_{2 N}=k \mid S_{2 n}=l\right)=\frac{h(l, 2 n, k, 2 N) P\left(S_{2 N}=k\right)}{\sum_{k^{\prime}=l}^{2 N-2 n+l} h\left(l, 2 n, k^{\prime}, 2 N\right) P\left(S_{2 N}=k^{\prime}\right)}
$$

In the case where the sample contains no defective tube, i.e. $l=0$, we have

$$
P\left(S_{2 N}=k \mid S_{2 n}=0\right)=\frac{P\left(S_{2 N}=k\right) h(0,2 n, k, 2 N)}{\sum_{k^{\prime}=0}^{2 N-2 n} P\left(S_{2 N}=k^{\prime}\right) h\left(0,2 n, k^{\prime}, 2 N\right)}
$$

Straigthforward algebraic manipulation using Eq.( 1) yields:

$$
h(0,2 n, k, 2 N)=\frac{(2 N-k) !(2 N-2 n) !}{(2 N) !(2 N-2 n-k) !}
$$

\subsubsection{One sample from one steam generator}

Let $S_{2 n}^{\prime}$ be the number of defective tubes in a random sample of size $2 n$ taken from steam generator nr 1 containing $N$ tubes. Hence, $S_{N}^{\prime}$ is the number of defective tubes in steam generator $\mathrm{nr} 1$.

In the case where the population of $2 N$ tubes is known to contain $k$ defective tubes, the probability of having $i$ defective tubes in steam generator $\mathrm{nr} 1$ is given:

$$
P\left(S_{N}^{\prime}=i \mid S_{2 N}=k\right)=\frac{\left(\begin{array}{c}
k \\
i
\end{array}\right)\left(\begin{array}{c}
2 N-k \\
N-i
\end{array}\right)}{\left(\begin{array}{c}
2 N \\
N
\end{array}\right)}=h(i, N, k, 2 N)
$$

If the population of $N$ tubes in steam generator $\mathrm{nr} 1$ is known to contain $i$ defective tubes, the probability of having $l$ defective tubes in a random sample of size $2 n$ taken from steam generator $\mathrm{nr} 1$ is given by 


$$
P\left(S_{2 n}^{\prime}=l \mid S_{N}^{\prime}=i\right)=\frac{\left(\begin{array}{l}
i \\
l
\end{array}\left(\begin{array}{c}
N-i \\
2 n-l
\end{array}\right)\right.}{\left(\begin{array}{l}
N \\
2 n
\end{array}\right)}=h(l, 2 n, i, N)
$$

Applying Bayes' theorem, the probability of $k$ tubes in the total population of $2 N$ tubes given that a random sample of size $2 n$ taken from steam generator $n r 1$ contains $l$ defective tubes is given by

$$
P\left(S_{2 N}=k \mid S_{2 n}^{\prime}=l\right)=\frac{P\left(S_{2 n}^{\prime}=l \mid S_{2 N}=k\right) P\left(S_{2 N}=k\right)}{P\left(S_{2 n}^{\prime}=l\right)}
$$

Applying the rule of total probabilities we have successively

$$
P\left(S_{2 n}^{\prime}=l\right)=\sum_{k=l}^{2 N-2 n+l} P\left(S_{2 n}^{\prime}=l \mid S_{2 N}=k\right) P\left(S_{2 N}=k\right)
$$

and

$$
P\left(S_{2 n}^{\prime}=l \mid S_{2 N}=k\right)=\sum_{j=l}^{k} P\left(S_{2 n}^{\prime}=l \mid S_{N}^{\prime}=i\right) P\left(S_{N}^{\prime}=i \mid S_{2 N}=k\right)
$$

Hence,

$$
P\left(S_{2 N}=k \mid S_{2 n}^{\prime}=l\right)=\frac{\sum_{i=l}^{k} P\left(S_{2 n}^{\prime}=l \mid S_{N}^{\prime}=i\right) P\left(S_{N}^{\prime}=i \mid S_{2 N}=k\right) P\left(S_{2 N}=k\right)}{\sum_{k^{\prime}=l}^{2 N-2 n+l} P\left(S_{2 N}=k^{\prime}\right) \sum_{i^{\prime}=l}^{k^{\prime}} P\left(S_{2 n}^{\prime}=l \mid S_{N}^{\prime}=i^{\prime}\right) P\left(S_{N}^{\prime}=i^{\prime} \mid S_{2 N}=k^{\prime}\right)}
$$

or

$$
P\left(S_{2 N}=k \mid S_{2 n}^{\prime}=l\right)=\frac{P\left(S_{2 N}=k\right) \sum_{i=l}^{k} h(l, 2 n, i, N) h(i, N, k, 2 N)}{\sum_{k^{\prime}=l}^{2 N-2 n+l} P\left(S_{2 N}=k^{\prime}\right) \sum_{i^{\prime}=l}^{k^{\prime}} h\left(l, 2 n, i^{\prime}, N\right) h\left(i^{\prime}, N, k^{\prime}, 2 N\right)}
$$

In the case where the sample contains no defective tube, i.e. $l=0$, we have

$$
P\left(S_{2 N}=k \mid S_{2 n}^{\prime}=0\right)=\frac{P\left(S_{2 N}=k\right) \sum_{i=0}^{k} h(0,2 n, i, N) h(i, N, k, 2 N)}{\sum_{k^{\prime}=0}^{2 N-2 n} P\left(S_{2 N}=k^{\prime}\right) \sum_{i^{\prime}=0}^{k^{\prime}} h\left(0,2 n, i^{\prime}, N\right) h\left(i^{\prime}, N, k^{\prime}, 2 N\right)}
$$

Again, a straightforward although somewhat tedious algebraic manipulation yields:

$$
\sum_{i=0}^{k} h(0,2 n, i, N) h(i, N, k, 2 N)=\frac{(2 N-k) !(2 N-2 n) !}{(2 N) !(2 N-2 n-k) !}
$$

This demonstrates the equality of Eq. (6) and Eq.( 15).

\subsubsection{Two samples from two steam generators}

Let $S_{n}^{\prime}$ and $S_{n}^{\prime \prime}$ be the number of defective tubes in two random samples, each of of size $n$, taken from steam generator $\mathrm{nr} 1$ and steam generator $\mathrm{nr} 2$ respectively. Each steam generator contains $N$ tubes. Hence, $S_{N}^{\prime}$ and $S_{N}^{\prime \prime}$ are the number of defective tubes in steam generator $\mathrm{nr}$ 1 and steam generator $\mathrm{nr} 2$ respectively. 
In the case where the total population is known to include $k$ defective tubes and the distribution of those defective tubes between steam generator $\mathrm{nr} 1$ ( $i$ defective tubes) and steam generator $\mathrm{nr} 2$ ( $k$-i defective tubes) is also known, we have

$$
P\left(S_{n}^{\prime}=l ; S_{n}^{\prime \prime}=m \mid S_{2 N}=k\right)=P\left(S_{n}^{\prime}=l \mid S_{N}^{\prime}=i\right) P\left(S_{n}^{\prime \prime}=m \mid S_{N}^{\prime \prime}=k-i\right)
$$

In the case where the distribution of the defective tubes between steam generators is unknown, application of the rule of total probabilities leads to

$$
\begin{aligned}
& P\left(S_{n}^{\prime}=l ; S_{n}^{\prime \prime}=m \mid S_{2 N}=k\right)= \\
& =\sum_{i=l}^{k} P\left(S_{n}^{\prime}=l \mid S_{N}^{\prime}=i\right) P\left(S_{n}^{\prime \prime}=m \mid S_{N}^{\prime \prime}=k-i\right) P\left(S_{N}^{\prime}=i \mid S_{2 N}=k\right)
\end{aligned}
$$

Applying Bayes' theorem, the probability of $k$ tubes in the total population of $2 N$ tubes given that random samples of size $n$ taken from steam generator $\mathrm{nr} 1$ and $\mathrm{nr} 2$ contain $l$ and $m$ defective tubes respectively is given by

$$
P\left(S_{2 N}=k \mid S_{n}^{\prime}=l ; S_{n}^{\prime \prime}=m\right)=\frac{P\left(S_{n}^{\prime}=l ; S_{n}^{\prime \prime}=m \mid S_{2 N}=k\right) P\left(S_{2 N}=k\right)}{P\left(S_{n}^{\prime}=l ; S_{n}^{\prime \prime}=m\right)}
$$

Applying again the rule of total probabilities, we have

$$
P\left(S_{n}^{\prime}=l ; S_{n}^{\prime \prime}=m\right)=\sum_{k=l+m}^{2 N-2 n+l+m} P\left(S_{n}^{\prime}=l ; S_{n}^{\prime \prime}=m \mid S_{2 N}=k\right) P\left(S_{2 N}=k\right)
$$

or

$$
\begin{aligned}
& P\left(S_{n}^{\prime}=l ; S_{n}^{\prime \prime}=m\right)= \\
& =\sum_{k=l+m}^{2 N-2 n+l+m} \sum_{i=l}^{k} P\left(S_{n}^{\prime}=l \mid S_{N}^{\prime}=i\right) P\left(S_{n}^{\prime \prime}=m \mid S_{N}^{\prime \prime}=k-i\right) P\left(S_{N}^{\prime}=i \mid S_{2 N}=k\right) P\left(S_{2 N}=k\right)
\end{aligned}
$$

Hence

$$
\begin{aligned}
& P\left(S_{2 N}=k \mid S_{n}^{\prime}=l ; S_{n}^{\prime \prime}=m\right)= \\
& =\frac{\sum_{i=l}^{k} P\left(S_{n}^{\prime}=l \mid S_{N}^{\prime}=i\right) P\left(S_{n}^{\prime \prime}=m \mid S_{N}^{\prime \prime}=k-i\right) P\left(S_{N}^{\prime}=i \mid S_{2 N}=k\right) P\left(S_{2 N}=k\right)}{2 N-2 n+l+m} \sum_{k^{\prime}=l+m}^{k^{\prime}} P\left(S_{n}^{\prime}=l \mid S_{N}^{\prime}=i^{\prime}\right) P\left(S_{n}^{\prime \prime}=m \mid S_{N}^{\prime \prime}=k^{\prime}-i^{\prime}\right) P\left(S_{N}^{\prime}=i^{\prime} \mid S_{2 N}=k^{\prime}\right) P\left(S_{2 N}=k^{\prime}\right) \\
& \text { or } \\
& P\left(S_{2 N}=k \mid S_{n}^{\prime}=l ; S_{n}^{\prime \prime}=m\right)= \\
& =\frac{P\left(S_{2 N}=k\right) \sum_{i=l}^{k} h(l, n, i, N) h(m, n, k-i, N) h(i, N, k, 2 N)}{2 N-2 n+l+m} P\left(S_{2 N}=k^{\prime}\right) \sum_{i^{\prime}=l}^{k^{\prime}} h\left(l, n, i^{\prime}, N\right) h\left(m, n, k^{\prime}-i^{\prime}, N\right) h\left(i^{\prime}, N, k^{\prime}, 2 N\right)
\end{aligned}
$$

In the case where both sample contain no defective tube, i.e., $l=0$ and $m=0$, we have

$$
\begin{aligned}
& P\left(S_{2 N}=k \mid S_{n}^{\prime}=0 ; S_{n}^{\prime \prime}=0\right)= \\
& =\frac{P\left(S_{2 N}=k\right) \sum_{i=0}^{k} h(0, n, i, N) h(0, n, k-i, N) h(i, N, k, 2 N)}{\sum_{k^{\prime}=0}^{2 N-2 n} P\left(S_{2 N}=k^{\prime}\right) \sum_{i^{\prime}=0}^{k^{\prime}} h\left(0, n, i^{\prime}, N\right) h\left(0, n, k^{\prime}-i^{\prime}, N\right) h\left(i^{\prime}, N, k^{\prime}, 2 N\right)}
\end{aligned}
$$

Again, a straightforward although somewhat tedious algebraic manipulation yields: 


$$
\sum_{i=0}^{k} h(0, n, i, N) h(0, n, k-i, N) h(i, N, k, 2 N)=\frac{(2 N-k) !(2 N-2 n) !}{(2 N) !(2 N-2 n-k) !}
$$

The equality of Eq. ( 6), Eq.( 15) and Eq.( 24) is therefore demonstrated. This leads to an expected conclusion: all the information obtained by the inspection of a random sample depends only on the size of the sample. It is therefore not important whether the random sample is taken from the total population or from a random partition of the total population. In the case of the steam generators, it is therefore not important whether the fixed size random sample is taken from one or both steam generators.

A useful practical consequence is that the numerical example in this paper could be fully described with minimized numerical effort by using equations developed in section 2.2.1. On the other hand, real life inspection of samples from both steam generators may be recommended. Such approach might, for example, reveal potential violations of our assumption that all steam generators perform in the same manner.

\subsubsection{Prior Distributions}

The choice of the prior distribution of defective tubes $P\left(S_{2 N}=k\right)$ is, as mentioned above, subjective. In the following, a few examples of prior distributions are discussed. They share a very useful feature: a closed-form posterior density.

In absence of any information it may be useful to consider a non-informative uniform prior distribution:

$$
P\left(S_{2 N}=k\right)=\frac{1}{2 N+1} \quad 0 \leq k \leq 2 N .
$$

Another practical choice of the prior distribution may be based on the reasonable expectation that small number of defective tubes is more likely than a large number. Assuming linear decrease of $P\left(S_{2 N}=k\right)$ with increasing $k$ then leads to:

$$
P\left(S_{2 N}=k\right)=\frac{2 N-k}{\frac{2 N}{2}(2 N+1)} \quad 0 \leq k \leq 2 N .
$$

We may also assume that the number of defective tubes in the finite population follows the binomial distribution:

$$
P\left(S_{2 N}=k\right)=\left(\begin{array}{c}
2 N \\
k
\end{array}\right) p^{k}(1-p)^{2 N-k} \quad 0 \leq k \leq N,
$$

with expected number of defective tubes in the finite population being $p 2 N$.

\subsection{Closed-Form Posterior Distributions}

The above priors applied in Eq. ( 6) lead to the closed-form posterior densities listed below. Additionally, closed-form solutions for expected number of defects left in the steam generator after a sampling inspection with zero defects and its variance are given. They are obtained using:

$$
\begin{aligned}
& <k>=\sum_{k=0}^{N} k P\left(S_{2 N}=k \mid S_{2 n}=0\right) \text { and } \\
& \operatorname{Var}(k)=\sum_{k=0}^{N}(k-<k>)^{2} P\left(S_{2 N}=k \mid S_{2 n}=0\right)
\end{aligned}
$$




\subsubsection{Non-informative uniform prior}

$$
\begin{gathered}
P\left(S_{2 N}=k \mid S_{2 n}=0\right)=\frac{1+2 n}{1+2 N} h(0,2 n, k, 2 N) \\
<k>=\frac{N-n}{1+n} \\
\operatorname{Var}(k)=\frac{N-n}{1+n} \quad \frac{(1+N)(1+2 n)}{(1+n)(3+2 n)} \\
P\left(S_{2 N}=0 \mid S_{2 n}=0\right)=\frac{1+2 n}{1+2 N}
\end{gathered}
$$

\subsubsection{Slightly informative triangular prior}

$$
\begin{aligned}
& P\left(S_{2 N}=k \mid S_{2 n}=0\right)=\frac{(2 N-k)\left(N+3 N n+2 N n^{2}\right)}{\left(N+2 N^{2}\right)(N+n+2 N n)} h(0,2 n, k, 2 N) \\
& <k>=\frac{(N-n)(2 n+2 N(1+2 n)-1)}{(3+2 n)(N+n+2 n N)} \\
& \operatorname{Var}(k)=\frac{2(1+N)(N-n)(1+2 n)\left(n+2 n^{2}+2 N^{2}(1+n)(1+2 n)+N(1+n)(6 n-1)\right)}{(2+n)(3+2 n)^{2}(N+n+2 n N)^{2}} \\
& P\left(S_{2 N}=0 \mid S_{2 n}=0\right)=\frac{N+3 N n+2 N n^{2}}{\left(\frac{1}{2}+N\right)(N+n+2 N n)}
\end{aligned}
$$

\subsubsection{Binomial prior}

$$
\begin{aligned}
& P\left(S_{2 N}=k \mid S_{2 n}=0\right)=\left(\frac{1}{1-p}\right)^{-2 N+2 n}(1-p)^{-k} p^{k}\left(\begin{array}{c}
2 N \\
k
\end{array}\right) h(0,2 n, k, 2 N) \\
& <k>=2 p(N-n) \\
& \operatorname{Var}(k)=2 p(N-n)(p-1) \\
& P\left(S_{2 N}=0 \mid S_{2 n}=0\right)=\left(\frac{1}{1-p}\right)^{-2 N+2 n}
\end{aligned}
$$

\subsubsection{Posterior (cumulative) distributions}

The posterior (cumulative) distributions are given by the following equations

$$
P\left(S_{2 N} \leq m \mid S_{2 n}=l\right)=\sum_{k=0}^{m} P\left(S_{2 N}=k \mid S_{2 n}=l\right)
$$

and

$$
P\left(S_{2 N}>m \mid S_{2 n}=l\right)=1-\sum_{k=0}^{m} P\left(S_{2 N}=k \mid S_{2 n}=l\right) .
$$

\subsection{Comments on the selection of the prior}

If we believe that the material and design improvements made in the replacement steam generators are so efficient that active degradation processes are unlikely and at the same time acknowledge that rare events might occur, a binomial distribution associated with a low value of the probability $p$ appears a well-suited distribution that describes correctly our prior information about the condition of the tubes. 
On the other hand, we may acknowledge the material and design improvements made in the replacement steam generators and at the same time remain convinced that the nature is more imaginative than the most experienced engineers. Than, our belief may well be that that no knowledge about the condition of the tubes exists prior to the inspection. In such situation, the uniform distribution may appear to be a well-suited distribution.

At the first glance, it might appear that the binomial distribution expresses more information about the actual proportion of defective tubes in the steam generators than the uniform distribution. However, the analysis of the resulting closed-form posteriors (see section 2.3) leads to different conclusion.

Some rearrangements of the posterior distribution with the binomial prior (Eq. (32)) lead to:

$$
P\left(S_{2 N}=k \mid S_{2 n}=0\right)=\left(\begin{array}{c}
2 N-2 n \\
k
\end{array}\right) p^{k}(1-p)^{2 N-2 n-k} \text {. }
$$

Now compare this result with binomial distribution (Eq. (28)). It is clear that the posterior in Eq. ( 35 ) is actually the prior we would have postulated for any subset of $2 N-2 n$ tubes in the population, without any data at all. Otherwise stated, the data collected during the inservice inspection of the first sample $(2 n)$ tells us nothing at all about the unsampled tubes $(2 N-2 n)$.

In other words, the binomial prior introduces a strong belief that there is a limited and rather well characterized subpopulation of defective tubes. Since we do not find any defective tubes during the inspection of the sample $2 n$, the entire defective subpopulation must have survived the inspection and remains in the uninspected set of the tubes. This indeed does not bring any new information about the uninspected tubes.

When postulating the uniform prior, the expected number $\langle k>$ of defective tubes in the population after that the inspection of the random sample of $2 n$ reveals zero defective tubes is given by Eq.(30). Then, the expected number of defective tubes left in the population is given by

$$
\frac{<k>}{2 N-2 n}=\frac{1}{2 n+2} \text {. }
$$

This leads to the conclusion that, when using a uniform prior, the data collected during the inservice inspection of a sample provide significant new information about the unsampled tubes. Indeed, for small samples, even a small increase in the sample size $n$ yields strong decrease in the expected number of defective tubes left undiscovered.

Such development corresponds well with our intuitive common-sense judgments: sampling inspection will only improve our knowledge about the defective subpopulation if we had very poor or no knowledge about it prior to the inspection.

\subsection{Approximate Solutions}

In the following, some approximate solutions obtained from the literature are outlined. They are primarily intended for comparison with closed-form solutions derived in this paper.

If $N$ is large enough and the sample size is small (say, $n \leq 0.05 N$ ), the binomial distribution can be used to approximate the hypergeometric distribution (Eq. ( 1)): 


$$
P\left(S_{n}=l \mid S_{N}=k\right)=h(l, n, k, N) \cong\left(\begin{array}{l}
n \\
l
\end{array}\right) p^{l}(1-p)^{n-l} \text { with } 0<\mathrm{p}<1
$$

where the probability of success $p$ per Bernouilli trial is $p=\frac{k}{N}$.

This approximation is powerful as it allows the use of prior-likelihood combinations called conjugate pairs, as discussed in [6] and in Section 2.1.

For example, the Beta distribution model is a conjugate prior for the proportion of successes $p$ when samples have a binomial distribution. In other words, the Beta family is conjugate to binomial data, that is, updating a Beta prior distribution with data produces a posterior that is also a Beta distribution.

If the parameter $k / N$ is assumed to be random and follows the Beta distribution with parameters $a$ and $b$, the prior distribution of $g(k / N)$ is

$$
g\left(\frac{k}{N}\right)=\frac{1}{B(a, b)}\left(\frac{k}{N}\right)^{a-1}\left(1-\frac{k}{N}\right)^{b-1} \text { with } a, b>0,
$$

By Bayes'theorem the posterior density of $p$, given the data $S_{n}=l$, is given by

$$
g\left(p \mid S_{n}=l\right)=\frac{1}{B(a+l, b+n-l)} p^{a+l-1}(1-p)^{b+n-l-1}, 0<\mathrm{p}<1 .
$$

The Bayes estimate of $p$ is given by the mean $<p>$ of the posterior distribution, i.e.,

$$
<p>=\frac{a+l}{a+b+n}
$$

If there is no defective tubes in the sample, i.e., $l=0$,

$$
g\left(p \mid S_{n}=0\right)=\frac{1}{B(a, b+n)} p^{a-1}(1-p)^{b+n-1}
$$

and the estimate $\langle p>$ for zero occurrence is

$$
<p>=\frac{a}{a+b+n}
$$

The selection of the beta distribution for the prior is interesting as the parameters $a$ and $b$ can be suitably selected to reflect prior opinion.

With $a=1, b=1$, the prior is flat and the estimate $\langle p>$ for zero occurrence reduces to

$$
<p>=\frac{1}{n+2} \text {. }
$$

Now, the Bayes estimate of the number of defective tubes, i.e., $<k>$ is

$$
<k>=<p>N=\frac{N}{n+2}
$$

If 5 percent of the 10,000 steam generators tubes are inspected and no defective tubes are found in the sample, the Bayes estimate of the number of defective tubes is $<k>=\frac{10,000}{500+2}=20$ defective tubes

This result is close to the expected mean value of defective tubes (Eq. (29)) i.e., 19 tubes. 
Another non-informative prior is the Jeffrey's prior and is given by the Beta distribution with $a=0.5$ and $b=0.5$. This prior corresponds roughly to the belief that either very few or almost all the tubes are defective. For this prior, the estimate of the number of defective tubes is

$<k>=\frac{N}{2(n+1)}=\frac{10,000}{2(501)}=10$ defective tubes

We can also consider an informative prior. To this end we need to choose values of $a$ and $b$. Suppose we have the belief that the expected number of defective tubes is about 2 and individual values range from 0 to 6 . This correspond to an expected value of $p=0.0002$ and $6 \sigma=0.0006$. Using the equations for the mean and the variance of the Beta distribution, the followings parameters a and $\mathrm{b}$ must be selected:

$a=4.0, b=19,991$

For this prior, the estimate of the number of defective tubes is

$<k>=\frac{4.00}{(4.00+19,991+500)} \times 10,000=2$ defective tubes

\section{NUMERICAL EXAMPLE}

In the numerical example we are investigating the inspection of steam generator tubes in a two-loop plant. Each of both steam generators is assumed to contain $N=5000$ tubes. The total finite population of tubes is therefore set to $2 N=10000$ in all subsequent discussions. Also, the definition of the relative sample size always refers to $2 N$. For example, a $10 \%$ sample would consist of 1000 tubes.

All calculations are based on closed-form posterior distributions outlined in section 2.3. These in turn are based on Eq.( 6), which assumes "one (common) sample from both steam generators" (section 2.2.1). Such approach has been shown as fully equivalent to other randomly selected samples (see sections 2.2.2 and 2.2.3), as long as both steam generators belong to the same population. The discussion is however not limited to this particular case: Eq.(6) is namely perfectly valid for a separate analysis of each of the steam generators.

\subsection{Uniform Prior}

The uniform prior distribution (Eq. ( 26)) assumes no a-priori knowledge on the number and distribution of defective tubes in the steam generators. In particular, any number of defective tubes is deemed to be equally probable. This results in the posterior density (Eq. (29)), which may be interpreted as the probability of having exactly $k$ defective tubes in the population $2 N$ after inspection of the random sample of size $2 n$ revealed zero defective tubes. Posterior densities for some selected inspection samples are plotted together with the prior density in Figure 1. 




Figure 1 Posteriors with different sample sizes with uniform prior and 0 defects found in the sample

The non-informative uniform prior (Eq. (26)) has a value of about $10^{-4}$, which is independent of the number of defective tubes in the steam generator. Now, assume that a inspection of a small $(0,5 \%)$ random sample has been performed without finding any defects in the sample. Our information about the uninspected tubes improved drastically (red curve in Figure 1): The probability of having small number of defects increased for about two orders of magnitude. As the same time, although not shown in Figure 1, the probability of having large number of defects also decreased significantly. The expected number of defective tubes, which was 5000 for the uniform prior, decreased to 191 (yellow dot on red curve in Figure 1).

Further increases in sample size are shown to increase the knowledge about the uninspected part of the population significantly. Inspecting the $20 \%$ random sample without founding any defects (magenta curve in Figure 1) for example results in expected number of defects at about 4 and in very fast decrease of probability of having larger numbers of defects.

Expected number of defective tubes in the uninspected part of the population is plotted as a function of the sample size in Figure 2. In addition, the $90 \%$ and $99 \%$ confidence curves are plotted, based on the expected number of degraded tubes and its variance defined in section 2.3.1. Without inspection, the expected number of defects is 5000. It diminishes fast with increasing the inspection sample. $3 \%$ inspection is shown to give $90 \%$ confidence, that there are less than 80 defective tubes left undetected. Similarly, 20\% inspection is shown to give $99 \%$ confidence, that there are less than 11 defective tubes left undetected. 


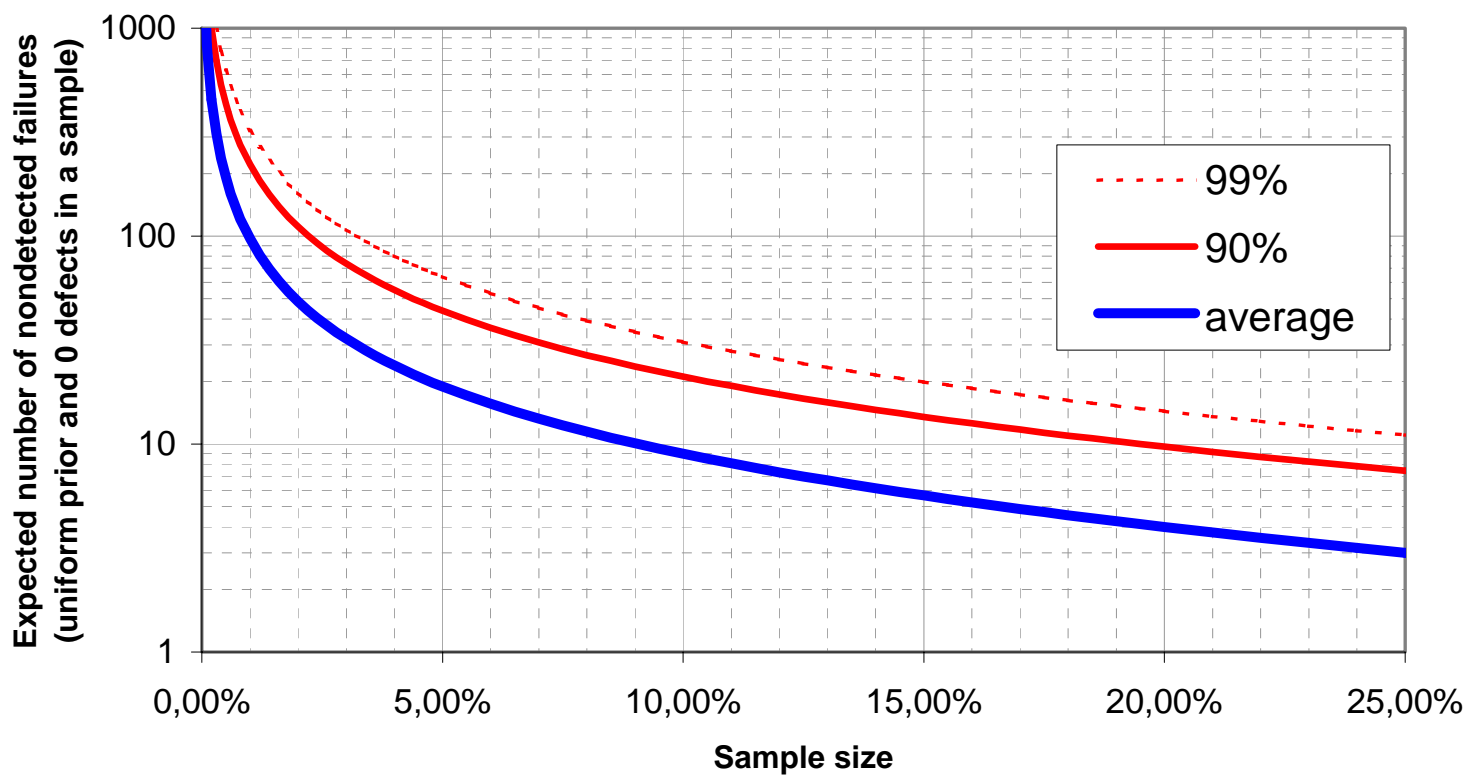

Figure 2 Expected number of undetected defective tubes with uniform prior and 0 defects found in the sample

\subsection{Triangular Prior}

The numerical example has been evaluated also using the triangular prior. The resulting probabilities were found to differ negligible from the probabilities obtained using the uniform prior and are not further discussed here. This is to some extent expected, since the numerical difference between Eq. (26) and (27) is rather small (in the order of $10^{-4}$ ).

\subsection{Binomial Prior}

The binomial prior distribution (Eq.( 28)) assumes rather specific a-priori knowledge on the number and distribution of defective tubes in the steam generators. An example with expected 40 defective tubes in both steam generators is plotted as the black curve in Figure 3. It is clear that the probability of having less than about 10 and more than about 80 defective tubes in both steam generators becomes exceedingly small.

The posterior distributions resulting from a set of assumed random sample sizes (Eq. ( 32)) are plotted together with the prior density in Figure 3. As stated in section 2.4, the information retrieved from the inspection of the random sample is rather weak, resulting in posteriors which are very close to the prior, the difference being only in the reduced size of the finite population.

Expected number of defective tubes in the uninspected part of the population is plotted as a function of the sample size in Figure 4. In addition, the $90 \%$ and $99 \%$ confidence curves are plotted, based on the expected number of degraded tubes and its variance defined in section 2.3.1. Without inspection, the expected number of defects is 40 . With increased sample size, it remains nearly constant: $3 \%$ inspection is shown to give $90 \%$ confidence, that there are less than about 105 defective tubes left undetected. Similarly, 20\% inspection is shown to give $90 \%$ confidence, that there are less than about 102 defective tubes left undetected. 




Figure 3 Posteriors with different sample sizes with binomial prior $(p=0.004)$ and 0 defects found in the sample

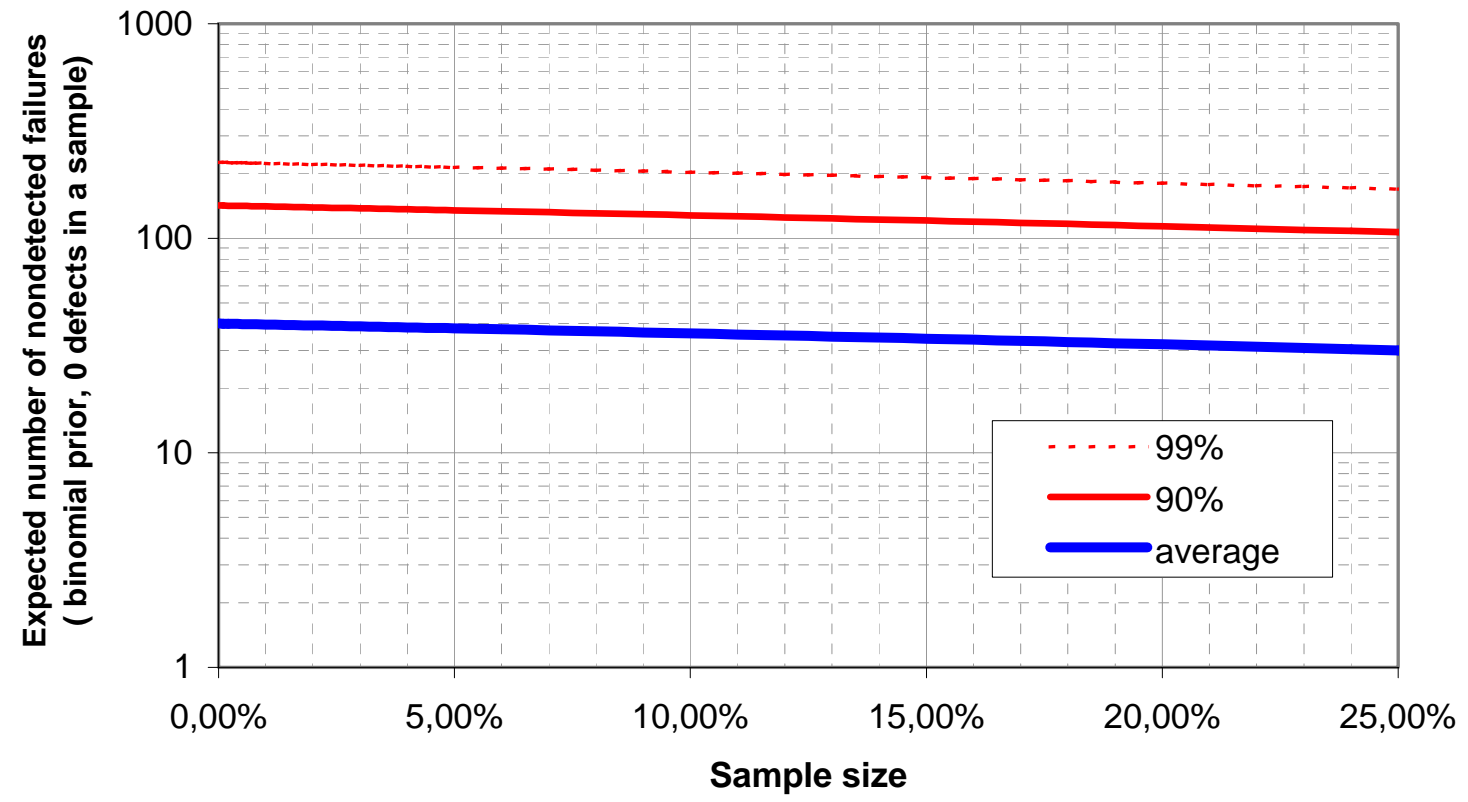

Figure 4 Expected number of undetected defective tubes with binomial prior $(p=0.004)$ and 0 defects found in the sample

\section{CONCLUSIONS}

The basis for determining the size of the random sample of tubes to be inspected in replacement steam generators is revisited in this paper. It is assumed that the probability of finding a defective tube in a random sample is exceedingly small. A procedure to estimate the maximum number of defective tubes left in the steam generator after no defective tubes have been detected in the randomly selected inspection sample is proposed. 
A Bayesian estimation is used to obtain closed-form solutions for uniform, triangular and binomial prior densities describing the number of failed tubes in steam generators. It is shown that the particular way of selecting the random inspection sample (e.g., one sample from both SG, one sample from each SG, etc.) does not affect the results of the inspection and also the information obtained about the state of the uninspected tubing, as long as the inspected steam generators belong to the same population.

Numerical examples are chosen to demonstrate two possible states of the knowledge existing before the inspection of the tubing. First, virtually no knowledge about the state of the steam generator tubing before the inspection is modeled using uniform and triangular prior densities. It is shown that the knowledge about the uninspected part of the tubing strongly depends on the size of the sample inspected. Further, even small inspection samples may significantly improve our knowledge about the uninspected part. Quantitative results for a typical batch of two steam generator with 5000 tubes each show that random inspection of about $3 \%$ of tubes with zero defects found indicates with $90 \%$ confidence that less than 80 defective tubes are left in the uninspected part of the tubing.

On the other hand, rather strong belief on the state of the tubing prior to the inspection is modeled using binomial prior density. In this case, the knowledge about the uninspected part of the tubing is virtually independent on the size of the sample. Furthermore, it is shown qualitatively and quantitatively that such inspection brings no additional knowledge on the uninspected part of the tubing.

The confidence to be placed in the results of sampling inspection therefore depends mainly on the knowledge about the defective tubes existing prior to the inspection. As a practical guide, the sampling inspection (with uniform prior) may be trusted as long as no defects are detected. With first failures detected, however, other inspection approaches might give more reliable results. This will be the topic of future investigations.

\section{REFERENCES}

[1] EPRI Report TR-107569-V2R5 (1998) PWR Steam Generator Examination Guidelines: Revision 5, Volume 2: Technical Basis.

[2] Roussel, G., Cizelj, L., Selection of Samples for Inservice Inspection of Steam Generator Tubes, ASME Pressure Vessels and Piping Conference, Denver, 2005.

[3] Cizelj, L., Roussel, G. Probabilistic Evaluation of Leak Rates Through Multiple Defects : the Case of Nuclear Steam Generators. Fatigue Fract. Eng. Mater. Struct., 2003, vol. 26, pp. 1069-1079.

[4] Cizelj, L., Mavko, B., Vencelj, P. Reliability of Steam Generator Tubes with Axial Cracks. J. Press. Vessel Technol., 1996, vol. 118, pp. 441-446.

[5] Dvoršek, T., Cizelj, L., Mavko, B. Safety and Availability of Steam Generator Tubes Affected by Secondary Side Corrosion. Nucl. Eng. Des.. 1998, vol. 185, pp. 11-21.

[6] Basu, A.P., Gaylor, D.W.and Chen, J.J. (1996) Estimating the probability of occurrence of tumor for a rare cancer with zero occurrence in a sample. Regulatory Toxicology and Pharmacology, 23, pp. 139-144.

[7] Scherwish, M. J., Theory of Statistics, Springer, 1997. 